l'esprit qu'il nous a semblé néanmoins intéressant de proposer aux lecteurs de $\mathrm{m} / \mathrm{s}$ tant est surprenante l'association d'une empreinte parentale de deux gènes codant pour un récepteur et son ligand, et si étrangement cohérente est cette association avec la théorie de Moore et Haig.

C.B.

A.K.

1. Babinet C, Barra J, Renard JP. L'indispensable complémentarité des génomes parentaux. médecine/sciences $1989 ; 5: 8-15$.

2. Paldi A, Jami J. L'empreinte génomique : complémentarité fonctionnelle des deux génomes parentaux. médecine/sciences 1991; 7 : 247-54.

3. Surani A, Reik W, Allen ND. Transgenes as molecular probes for genomic imprinting. Trends Genet 1988 ; 4 : 59-62.

4. Barlow DP, Stöger R, Herrmann BG, Sailo K, Schweifer N. The mouse insulin-like growth factor type 2 receptor is imprinted and closely linked to the tme locus. Nalure 1991 ; 349: 84-87.

5. De Chiara TM, Efstratiadis A, Robertson EL. A growth-deficiency phenotype in heterozygous mice carrying an insulin-like growth factor II gene disrupted by gene targeting. Nalure 1990 ; $345: 78-80$.

6. Moore T, Haig D. Genomic imprinting in mammalian development : a parental tug-ofwar. Trends Genet 1991; 7 : 45-9.

Note ajoutée aux épreuves

L'existence d'une empreinte parentale du gène $I G F-2$ est maintenant bien établie. [De Chiara TM et al. Cell $1991 ; 64$ : 849-60].

\title{
Une lésion moléculaire sur le chromosome 21 dans la maladie d'Alzheimer
}

L'annonce de la découverte d'une lésion moléculaire dans la maladie d'Alzheimer par l'équipe dirigée par T. Hardy et R. Williamson [1] a fait sensation, car c'est la première fois qu'une anomalie précise est décrite dans cette affection. Il importe de replacer cette avancée dans le cadre général de la maladie, afin de déterminer dans quelle mesure doivent changer les concepts destinés à orienter désormais la recherche dans un domaine dont $\mathrm{m} / \mathrm{s}$ a parlé à de nombreuses reprises*.

On sait que dans le cerveau des sujets atteints de maladie d'Alzheimer s'accumule un peptide de 42 acides aminés au niveau des plaques et des pelotons neurofibrillaires. Ce peptide dit A4 ou $\beta$-amyloïde dérive d'une protéine appelée précurseur de la protéine amyloïde ou APP ; celui-ci se présente sous plusieurs formes, provenant d'épissages différentiels du même gène, dont les principales sont appelées suivant le nombre d'acides aminés de leur séquence, APP 695, 751 ou 770 ; les deux dernières possédant une séquence dotée des propriétés d'un inhibiteur de protéases $\left(\mathrm{m} / \mathrm{s} n^{\circ} 5\right.$, vol. 4, p. 323). Le gène de l'APP est situé sur le chromosome 21.

L'APP a été directement mise en cause dans une autre maladie avec dépôts amyloïdes, l'hémorragie cérébrale avec amyloïdose $\left(\mathrm{m} / \mathrm{s} n^{\circ} 7\right.$, vol. 6, p. 704). Cette observation a contribué à inciter Goate et al. [1], une équipe anglo-américaine constituée de 19 signataires, à analyser

\footnotetext{
* Articles ou nouvelles parues antérieurement dans $\mathrm{m} / \mathrm{s}$ concernant la maladie d'Alzheimer: $n^{\circ} 5$, vol. 3, p. 254 et $256 ; n^{\circ} 10$, vol. 3, p. $620 ; n^{\circ} 5$, vol. 4, p. 323 : n० 9, vol. 5, p. 689 el 690 ; $n^{\circ} 3$, vol. 6, p. $312 ; n^{\circ} 6$, vol. 6, p. $602 ; n^{\circ} 9$, vol. 6, p. 923 .
}

l'ADNc de l'APP 770 en employant avant tout la méthode d'amplification de l'ADN dite PCR. Ils se sont adressés à des formes familiales et ont concentré leurs efforts sur une famille anglaise dont deux frères avaient épousé deux sœurs. Chez les malades, et eux seuls, ils ont trouvé une mutation ponctuelle unique, au nucléotide 2149 de l'ADNc, entraînant un remplacement d'une valine par une isoleucine. Cette mutation se plaçait deux acides aminés en aval par rapport à l'extrémité C-terminale du peptide amyloïde, en position 717 , donc à l'intérieur de la membrane (figure 1). Bien que la modification Val-Ile soit dite conservatrice (elle ne change pas la charge et peu la conformation), le résultat prévisible de cette substitution est d'augmenter l'hydrophobicité du domaine transmembranaire et de renforcer l'ancrage de l'APP à la membrane. Ainsi se comprendrait la rupture de la molécule protéique au niveau de l'extrémité C-terminale du peptide amyloïde, ce qui ne semble pas être le processus normal de dégradation de l'APP $\left(\mathrm{m} / \mathrm{s} n^{\circ} 6\right.$, vol. 6, p. 602). La même mutation a été trouvée dans une autre famille, américaine, et non apparentée à la première. Comme elle ne l'a pas été dans 17 autres familles examinées, dont l'anomalie était pourtant liée au chromosome 21, une hétérogénéité génétique de la maladie d'Alzheimer apparaît évidente, même dans les formes familiales. Cette hétérogénéité était déjà connue, car elle était le seul élément indiscutable qui ressortait d'une vaste enquête portant sur 48 familles et dont $\mathrm{m} / \mathrm{s}$ a rendu compte en novembre 1990 ( $n^{\circ} 9$, vol. 6, p. 923). L'hétérogénéité peut se situer à deux niveaux. 
(a) Niveau chromosomique : beaucoup de formes familiales, surtout celles d'origine "précoce" (avant 65 ans) paraissent liées à une anomalie du chromosome 21 ; les formes tardives et celles qui ne sont pas familiales ne lui sont pas liées.

(b) Niveau du locus : les premières tentatives de localisation, en 1987, assignaient déjà un locus de forme familiale au chromosome 21 ; cependant plusieurs enquêtes approfondies (m/s $n^{\circ} 10$, vol. 3, p. 620) semblaient avoir éliminé toute possibilité d'intervention du gène $A P P$, du fait de recombinaisons qui faisaient placer ce gène à au moins 10 centimorgans de celui de la maladie d'Alzheimer. Les résultats actuels obligent à repenser les relations génétiques de la maladie, mais ne permettent pas de nier les données d'il y a quatre ans. On pourrait supposer qu'il existerait des cas dus à une altération qualitative de l'APP et d'autres qui ne le seraient pas. Il pourrait aussi exister des hyperproductions de l'APP, soit par dosage génique, soit par anomalie de facteurs de régulation. C'est ainsi que dans la trisomie 21, l'appa-

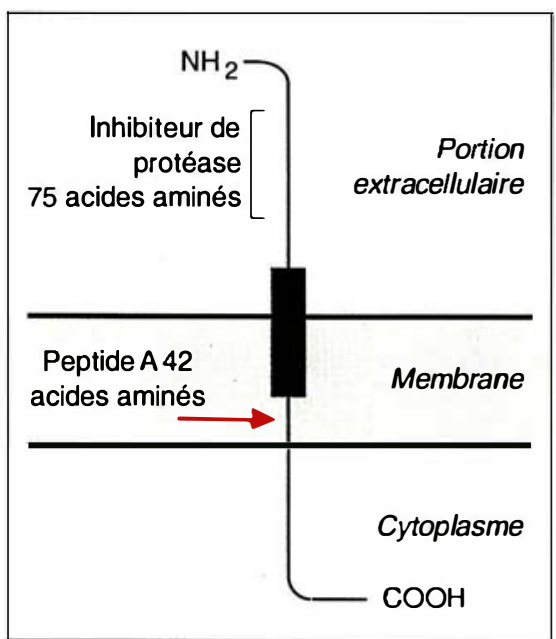

Figure 1. Schéma de I'APP 770. Le peptide A4 est représenté par le rectangle noir. La flèche (en rouge) indique le point de mutation. La taille des différents segments de la protéine n'est pas à l'échelle (modifié d'après $\mathrm{m} / \mathrm{s} \mathrm{n}^{\circ} 9$, vol. 5 , p. 690).

$\mathrm{m} / \mathrm{s} n^{\circ} 3$, vol. 7 , mars 91 rition précoce de syndromes du type Alzheimer est fréquente.

Une hyperproduction d'APP peut aussi être obtenue en culture dans certaines conditions expérimentales, par transfection de cellules COS par l'ADNc de l'APP [2] ; et, récemment, une équipe californienne [3] a observé que, dans les cultures de fibroblastes sénescents, on voit augmenter l'expression de l'APP, ce qui pourrait être en rapport avec l'accumulation de protéine $\beta$-amyloïde dans le cerveau de sujets atteints de la forme sénile, non familiale, de maladie d'Alzheimer. On pourrait ainsi concevoir, par analogie avec l'effet de certains oncogènes, deux façons différentes pour la protéine APP d'exercer des effets néfastes : un effet qualitatif, une mutation empêchant la dégradation normale et ancrant le peptide $\beta$-amyloïde dans la membrane ; un effet quantitatif, la surproduction ne permettant pas à l'excès d'APP de subir son catabolisme normal et entraînant des coupures transmembranaires inhabituelles.

On le voit, la découverte de Goate et al. marque une étape importante dans la connaissance des mécanismes moléculaires de la maladie, tout en soulevant pour l'avenir de redoutables problèmes, notamment celui de savoir s'il est utile de reconnaître à l'avance une maladie dont les conséquences sont sévères mais tardives. Il reste à déterminer la fréquence de cette mutation, ou d'autres dans le même gène. Enfin, pour prouver que c'est bien cette mutation qui est la cause du syndrome clinique, on se propose de fabriquer des souris transgéniques possédant le gène ou l'ADNc muté. Mais quel délai exigeront ces souris transgéniques pour manif ester les éventuels symptômes pathologiques? En attendant, on peut tenter la même expérience par transfection in vitro, dans l'espoir d'observer des modifications du catabolisme de l'APP.

J.C.D.
1. Goate A, Chartier-Harlin MC, Mullan M et al. Segregation in a missense mutation in the amyloid precursor gene with familial $\mathrm{Alz}$ heimer's disease. Nalure 1991; 349 : 704-6. 2. Maruyama K, Terakado $K$, Usami $M$, Yoshikawa K. Formation of amyloid like fribrils in COS cells overexpressing part of the Alzheimer amyloid protein precursor. Nalure 1990 ; 347 : 566-9.

3. Adler MJ, Coronel C, Shelton E, Seegmiller JE, Dewji NN. Increased gene expression of Alzheimer disease $\beta$-amyloid precursor protein in senescent cultured fibroblasts. Proc Nall Acad Sci USA 1991; 88 : 16-20.

INDEX
DES NOUVELLES
BREVES
L'interféron $\alpha$ active la phospho-
lipase A2 et la production d'acide
arachidonique (page 287).
Un gène pour le syndrome de Tur-
ner: une piste à suivre (page 289).
Pouvoir transformant des gènes
homéotiques (page 290).
Inactivation par double recombinai-
son homologue des deux allèles du
gène pim-1 (page 290).
Déficit partiel du transporteur de
glucose GLUT-2 dans un modèle
animal de diabète non insulino-
dépendant (page 291).
Contrôle du sexe de l'enfant à nai-
tre (page 291).
Un ADN monocaténaire détecté par
microscopie électronique dans le
cerveau d'animaux atteints de
tremblante (page 291).
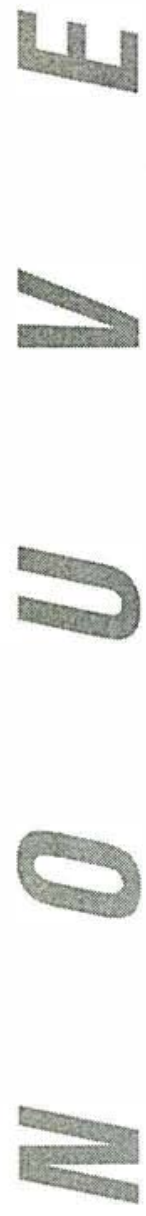